\title{
CAVITY-POLARITON EFFECTS IN II-VI MICROCAVITIES
}

\author{
R. André, F. Boeuf, D. Heger; Le Si Dang, R. Romestain \\ Laboratoire de Spectrométrie Physique, CNRS UMR 5588, Université J. Fourier \\ Grenoble 1, B.P. 87, 38402 Saint Martin d'Hères Cedex, France
}

\section{J. Bleuse AND M. MÜLleR}

CEA-Grenoble, DRFMC/SP2M/PSC

17 rue des Martyrs, 38054 Grenoble Cedex 9, France

\begin{abstract}
Semiconductor microcavities are monolithic multilayer heterostructures grown by molecular beam epitaxy. They allow the confinement along the growth axis of both photons between the Bragg reflectors and excitons in quantum wells. If the exciton-photon coupling matrix element is large enough compared to the line width, the system is said to be in the strong coupling regime. In that case a quantum well exciton couples to another discrete state: the photon mode of a planar microcavity with the same in-plane wave vector, to give rise to quasi-stationary states named cavity polaritons. In this regime, the Fermi golden rule does not hold any more and the optical properties, linear or nonlinear, are strongly related to polariton features. A review of the optical properties of CdTe-based microcavities operating in the strong coupling regime is given in this paper. The strength of the exciton-photon coupling, dynamic optical properties, and relaxation processes along polariton dispersion curves will be discussed, as well as stimulated emission of cavity polariton luminescence.
\end{abstract}

PACS numbers: 71.36.+c, 42.55.Sa, 78.66.-w

\section{Introduction}

Semiconductor microcavities are very interesting tools for the fundamental study of light-matter interaction in semiconductors. An important step in the physics of semiconductors was to confine excitons, first in quantum wells and then in quantum wires or quantum dots. These systems with reduced dimensionality allow an increase in oscillator strength and modification of electronic density of states, but they do not affect light. In quantum well microcavities (QMCs), both excitons and photons are controlled by confinement. A microcavity may be considered as a one-dimensional photonic band gap material where photons are free to propagate only in two dimensions. The excitons confined in quantum wells are 
free in the same plane as the cavity photons. This situation leads to the formation of cavity polaritons who are 2D analogues of polaritons in bulk semiconductors. It.will be shown in this paper that cavity polariton effects are determinant for the optical properties of QMCs.

QMCs are designed to confine photons and excitons along the growth direction. For the photon confinement, a microcavity consists of two highly reflecting mirrors, namely the Bragg reflectors, separated by a fine spacer whose optical thickness is a small integer number of $\lambda / 2$, where $\lambda$ is the wavelength of the optical transition of interest. Inside the cavity the amplitude of the stationary electromagnetic field exhibits a few nodes and antinodes, where quantum wells could be located to have respectively a vanishing or maximum coupling with light. If the exciton-photon coupling matrix element is large enough, as compared to the exciton or cavity mode line width, the system is in the strong coupling regime. In that case a quantum well exciton can couple only to another discrete state: the photon mode of the planar microcavity with the same in-plane wave vector to give rise to mixed quasi-stationary states: cavity polaritons. In a microcavity, quantum well (QW) excitons are not coupled to a continuum of photon states like in free space and consequently the Fermi golden rule does not hold any more. This situation is often referred to as the non-perturbative regime: the system oscillates reversibly between its excitonic and photonic nature only limited in time by leakage of the photon outside the cavity through imperfect mirrors or nonradiative scattering of the exciton.

As suggested above, the optical properties of QMCs are strongly related to polariton features. The aim of this paper is to give an overview of experimental results on cavity polaritons obtained in the particular case of our CdTe-based samples. The existence of cavity polaritons has been first evidenced in III-V materials [1], but the exciton-photon coupling and related effects are remarkably stronger in II-VI semiconductors thanks to their higher excitonic oscillator strength. Hence, II-VI samples are well suited to study the physics of cavity polaritons. Three main aspects of cavity polaritons will be discussed here. First, reflectivity measurements at normal incidence and low intensity evidence the strong coupling and are used to probe the exciton oscillator strength and exciton nonradiative broadening versus temperature. Second, photoluminescence (PL) dynamics measurements at low temperature and at non-resonant excitation are performed to study relaxation along the lower polariton dispersion branch via emission of acoustic phonons. A "bottleneck" effect is evidenced in the relaxation phenomena. Finally, we discuss stimulated emission of cavity polariton under high non-resonant excitation: a stimulation of the lower polariton PL is observed for an excitation density well below screening of excitons. The mechanisms of stimulation are very different from those of the usual lasing effect as in vertical cavity surface emitting laser.

\section{General properties of quantum well microcavities}

\subsection{Cavity polariton physics}

In 1994-1995, two years after the first experimental evidence of QMC normal mode splitting, several theoretical papers reported on the Rabi splitting in 
QMC and cavity polariton dispersion [2] with fully quantum-mechanical calculation [3], semiclassical [4], or both classical and quantum-mechanical approach [5]. Recently, very interesting review articles were published on the physics of strong coupling phenomena [6] and optical properties of microcavity polaritons [7]. The main points will be briefly summarized here, but a complete comprehension of the physics of cavity polaritons requires a careful reading of the above references.

In $\mathrm{QMC}$, the two states which couple are exciton and photon states. The QW material band gap and confinement effects determine the minimum exciton energy $E_{\mathrm{X}}(0)$ and the kinetic energy for the free motion, in the QW plane, of the exciton center-of-mass is given by the effective mass $M: E_{\mathrm{X}}\left(k_{\|}\right)=E_{\mathrm{X}}(0)+$ $\hbar^{2} k_{\|}^{2} / 2 M$. The cavity mode energy differs from the photon energy in free space by the fact that the wave vector is quantized along the growth direction: $E_{\mathrm{Ph}}\left(k_{\|}\right)=$ $\left(\hbar c / n_{\text {cav }}\right) \sqrt{\left(2 \pi / L_{\text {cav }}\right)^{2}+k_{\|}^{2}}$. The photon dispersion curve is parabolic at small $k_{\|}$ and may be associated to an effective mass by developing $E_{\mathrm{Ph}}$ to the first order in $k_{\|}^{2}$. The photon mass is $10^{4}$ to $10^{5}$ times smaller than the exciton effective mass, therefore the exciton dispersion is often negligible in polariton calculation. An exciton at wave vector $k_{\|}$can couple, by dipolar electric interaction, to a photon with the same in-plane vector. The eigenvalues of the coupling Hamiltonian $H$ give the eigenenergies of the system and the eigenstates are the cavity polaritons

$$
H=\left(\begin{array}{cc}
E_{\mathrm{cav}}-\mathrm{i} \Gamma_{\mathrm{cav}} & V \\
V & E_{\mathrm{X}}-\mathrm{i} \Gamma
\end{array}\right) .
$$

Imaginary parts are added to the energies to take bare states broadening into account. The polariton energies are then (see examples in Fig. 1):

$$
\begin{aligned}
& E_{\mathrm{pol} \pm}=\left[E_{\mathrm{X}}+E_{\text {cav }}-\mathrm{i}\left(\Gamma+\Gamma_{\text {cav }}\right)\right] / 2 \\
& \quad \pm \sqrt{V^{2}+\left[E_{\mathrm{X}}-E_{\text {cav }}-\mathrm{i}\left(\Gamma-\Gamma_{\text {cav }}\right)\right] / 4} .
\end{aligned}
$$

The dispersion curves of these two cavity polariton states are usually referred to as lower and upper polariton branch (LPB and UPB). At exciton-photon resonance the polariton states splitting, or the Rabi splitting, reads $\hbar \Omega_{\text {Rabi }}=$ $2 \sqrt{V^{2}-\left(\Gamma-\Gamma_{\text {cav }}\right)^{2} / 4}$. If $4 V^{2} \leq\left(\Gamma-\Gamma_{\text {cav }}\right)^{2}$, the two polaritons are degenerate at the bare states energy and the coupling affects only broadening. This situation is the weak coupling regime.

In the simple case of two identical mirrors (with reflectivity $R$ ) and one QW of vanishing thickness located at an antinode of the confined electromagnetic field, the coupling matrix element is given by

$$
V^{2}=\frac{c \Gamma_{0}}{n_{\text {cav }} L_{\text {eff }}} \frac{1+\sqrt{R}}{2 \sqrt{R}}
$$

The bare exciton radiative line width $\Gamma_{0}$ is proportional to the 2D exciton oscillator strength per surface unit $f_{x y}$. $L_{\text {eff }}$ is an effective cavity length corrected to include the penetration depth of the electromagnetic field into dielectric mirrors and resulting phase shift at the cavity mirror interfaces. It is interesting to note that for large coupling matrix element (compared to line width), the following simple relation holds: $\hbar \Omega_{\mathrm{Rabi}}=2 V \propto \sqrt{N f_{x y}}$, where $N$ is the number of QWs located 

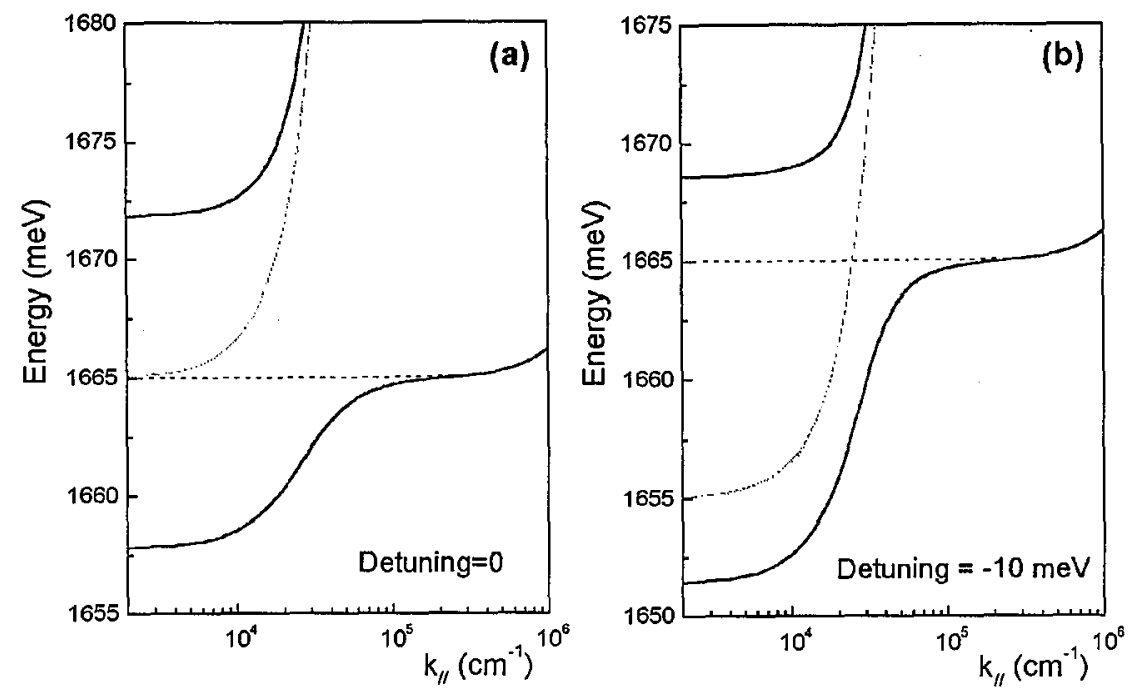

Fig. 1. Example of cavity-polariton dispersion curves calculated at resonance (a) or for a detuning $\Delta E=-10 \mathrm{meV}$ (b). Dashed and dotted curves are respectively the dispersion of bare exciton and photon states.

exactly at an antinode of the confined electromagnetic stationary field (otherwise an effective QW number smaller than $N$ has to be introduced).

The two coupled oscillator model is not just an intuitive description of cavity polariton. It can be derived rigorously from the complete quantum-mechanical calculation with the only assumption of being close to resonance [7]. In the case of simple QMC, this model gives analytic expressions for the description of strong coupling and cavity polaritons. It is very useful to understand the effects of the different physical parameters of the problem, but it includes approximations and is not suitable to spectra calculation. For the purpose of sample design, spectra modeling, and precise interpretation of the Rabi splitting in terms of excitonic oscillator strength, we performed numerical calculation using the transfer matrix formalism to describe a multilayer sample, including QWs. The optical properties of each layer (propagation and reflections at the interfaces) are modeled by a matrix acting on the basis of planar waves traveling forward and backward along the growth axis [8]. Moreover, it has been shown that the 2D exciton-radiation interaction in QWs may be treated in a semiclassical theory $[4,9,10]$ : the optical response of an excitonic resonance in a $Q W$ is described as a plane reflector whose transfer matrix $T_{\mathrm{QW}}$ for a $\mathrm{QW}$ exciton, at resonant frequency $\omega_{0}$ is

$$
T_{\mathrm{QW}}=\frac{1}{t}\left(\begin{array}{cc}
t^{2}-r^{2} & r \\
-r & t
\end{array}\right) \text { with } t=1+r \text { and } r=\frac{\mathrm{i} \Gamma_{0}}{\omega_{0}-\omega-\mathrm{i}\left(\Gamma_{0}+\gamma\right)} .
$$

$\Gamma_{0}$ is the radiative width of the exciton line and $\gamma$ is the nonradiative exciton broadening $\left(\Gamma=\Gamma_{0}+\gamma\right)$. To take into account the finite thickness $L_{\mathrm{w}}$ of the QW, $t$ and $r$ have to be multiplied by the propagation factor $\exp \left(\mathrm{i} k_{z} L_{\mathrm{w}}\right)$. Reflectivity, transmission and absorption properties of the sample are deduced from the transfer matrix of the whole sample and boundary conditions. For a more real- 
istic description of the optical properties of a layered structure and optimization of sample growth and design, we included in the calculation some sample defects such as losses due to diffusion of light by interface roughness [11], inhomogeneous broadening of exciton lines, effects of growth rate fluctuations or drift. Excitonic parameters have been first taken in the literature [12] for preliminary calculation and then adjusted by fitting our experimental results. The dispersion of refractive indices of CdMnTe and CdMgTe alloys at low temperature are the results of a preliminary work published in Ref. [13].

\subsection{Sample description and growth details}

A typical II-VI QMC structure is schematically drawn in Fig. 2. The mirrors are Bragg reflectors consisting of periodic stacks of $\mathrm{Cd}_{0.4} \mathrm{Mg}_{0.6} \mathrm{Te} / \mathrm{Cd}_{0.75} \mathrm{Mn}_{0.25} \mathrm{Te}$ $\lambda / 4$ layers. The typical number of periods is about 20 with a few periods more on the substrate side and a few periods less in the top mirror. This choice of alloy compositions is a compromise for telluride materials: the vanishing lattice mismatch between $\mathrm{Cd}_{0.40} \mathrm{Mg}_{0.60} \mathrm{Te}$ and $\mathrm{Cd}_{0.75} \mathrm{Mn}_{0.25} \mathrm{Te}$ avoids strain-induced dis-

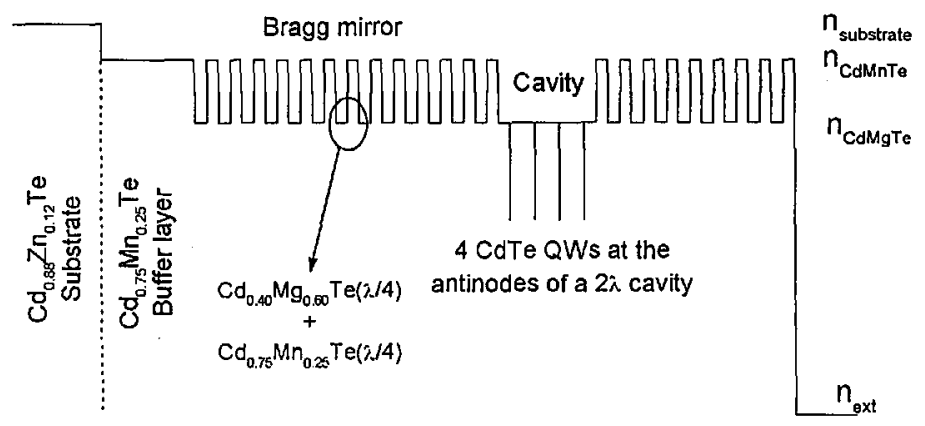

Fig. 2. Sketch of a CdTe-based microcavity sample on a refractive index scale. The number of periods in the Bragg reflectors was reduced for clarity (typical values are 20 pairs for the substrate side mirror and 15 for the front mirror).

locations in thick structure and their refractive index modulation $(\Delta n / n \approx 13 \%$ just above $\mathrm{CdTe}$ band gap) is large enough to build interferential mirrors. Higher magnesium concentrations would give slightly higher index modulation but the sample lifetime could be dramatically shortened by uncontrolled oxidation processes in $\mathrm{Cd}_{1-x} \mathrm{Mg}_{x} \mathrm{Te}(x \geq 0.7)$. CdTe QWs ( 5 to $8 \mathrm{~nm}$ thick) are embedded in the mirrors spacer whose thickness is $\lambda / 2, \lambda, 3 \lambda / 2$, or $2 \lambda$. The number of $Q W s$ per sample varies from 1 to 16 to explore various Rabi splittings and exciton states degeneracy. The barriers between adjacent wells are always thick enough to avoid overlap of carriers envelope functions.

Our samples are monolithically grown by conventional molecular beam epitaxy on (100)-oriented $\mathrm{Cd}_{0.88} \mathrm{Zn}_{0.12} \mathrm{Te}$ substrates. The different layers are grown under excess of $\mathrm{Cd}$ flux at about $300^{\circ} \mathrm{C}$. Since errors on layer thickness are directly proportional to the wavelength of the Bragg reflectors stop-band center and of the cavity mode, growth rates have to be very stable and carefully calibrated. The 
growth rate (one monolayer per second) is monitored by the oscillations of the reflection high-energy electron diffraction (RHEED) specular spot intensity. During about 8 to $9 \mathrm{~h}$ of sample growth, the growth rate is conserved within $\pm 0.5 \%$. There is no rotation of the substrate during the growth and the substrate is placed off the effusion cells axis to get a linear thickness gradient of about $7 \%$ along our $1 \mathrm{~cm}$ long samples. This gradient allows the cavity mode energy tuning with respect to the QW exciton by moving the observation spot. Interface roughness is also a point to control to avoid photon diffusion which would decrease photon lifetime inside the cavity. This was achieved by growth interruptions under $\mathrm{Cd}$ flux at each interface.

\section{Experimental investigation of the strong coupling regime}

\subsection{The Rabi splitting and oscillator strength}

The simplest way to evidence the strong coupling regime is to observe the anticrossing between exciton and photon lines in real space, at $k_{\|}=0$, for example on reflectivity spectra at normal incidence. For this purpose a series of reflectivity spectra were taken at successive positions along a wedge-shaped sample (Fig. 3). This sample (S6) contains $6 \mathrm{CdTe} / \mathrm{Cd}_{0.8} \mathrm{Mn}_{0.2} \mathrm{Te} \mathrm{QWs}(50 \AA$ thick) embedded in a $3 \lambda / 2$ cavity. An anticrossing behavior is observed when the cavity mode is brought into resonance with different excitonic levels whose energy is almost constant. This effect is clearly demonstrated in Fig. 4 where the energies of the different polariton lines are plotted versus the low energy side lobe of the stop-band which is used as a reference for sample thickness. The result is a complex polariton system with three coupling frequencies: the cavity mode couples with the ground state exciton $\mathrm{E} 1 \mathrm{H} 1(1 s)$ and to two excited levels identified as E1H1(2s) and E1L1(1s), respectively, at $22 \mathrm{meV}$ and $41 \mathrm{meV}$ above the ground state.

In a good approximation, valid when line widths are small compared to the coupling matrix element, the Rabi splitting for a cavity polariton varies as the square root of the exciton oscillator strength. Within this approach, the derivation of relative oscillator strengths is straightforward: the oscillator strength of the $2 s$ heavy hole exciton is found to be about $6.6 \%$ of that of the $1 s$ level. Absolute values and more accurate comparison were derived from spectra calculation with the formalism depicted previously: by fitting the calculated spectra to the experimental one, we obtain for each exciton the radiative line width $\Gamma_{0}$ which is proportional to the oscillator strength per unit area [4]: $f_{x y}=\Gamma_{0}\left(4 \varepsilon_{0} n_{\text {cav }} m_{0} c\right) / e^{2}$ ( $e$ is the electron charge and $m_{0}$ - the free electron mass). The result for $\operatorname{E} 1 \mathrm{H} 1(1 \mathrm{~s})$ is $2.3 \times 10^{13} \mathrm{~cm}^{-2}$ (in good agreement with absorption measurements in CdTe-based QWs [12] and about one order of magnitude higher than in III-V heterostructures [14]). For $\mathrm{E} 1 \mathrm{H} 1(2 s)$ and $\mathrm{E} 1 \mathrm{~L} 1(1 s)$ we obtain $1.5 \times 10^{12}$ and $1.1 \times 10^{13}$, respectively. Those results were found to be in very good agreement with exciton envelope function calculation [15].

The same method was applied successfully on a $p$-type modulation doped CdTe QWs inserted in a microcavity to study the oscillator strength transfer from the free exciton $\mathrm{X}$ to the positively charged trion $\mathrm{X}^{+}$when the $2 \mathrm{D}$ hole density 


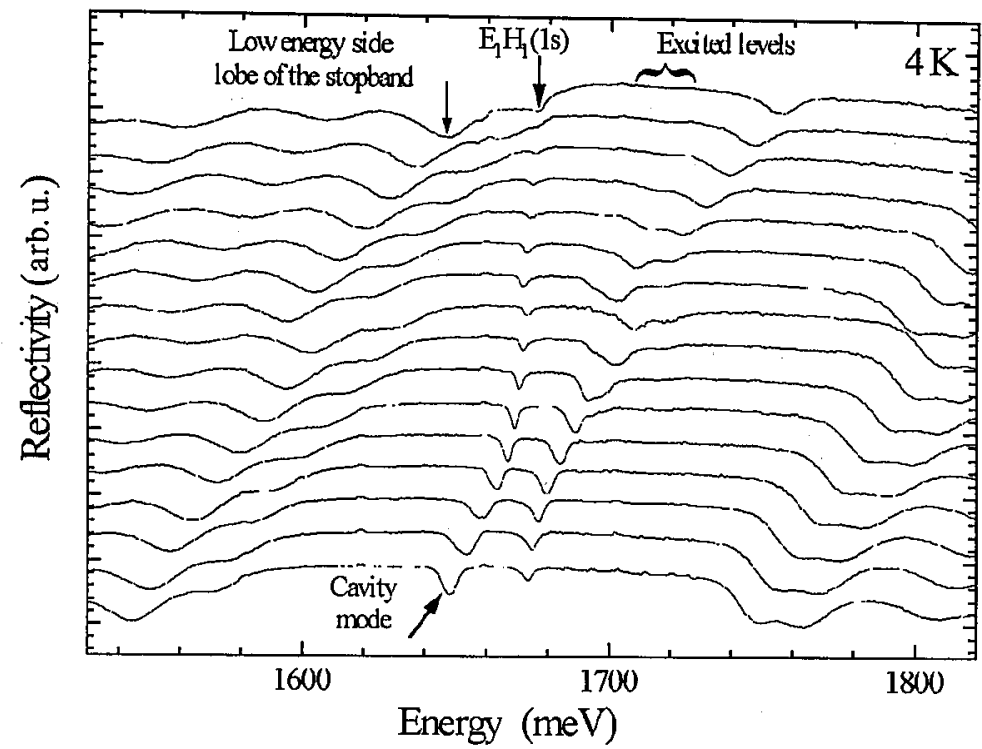

Fig. 3. Low temperature reflectivity spectra taken at successive positions along a wedge-shaped sample. The cavity mode anticrosses excitonic levels.

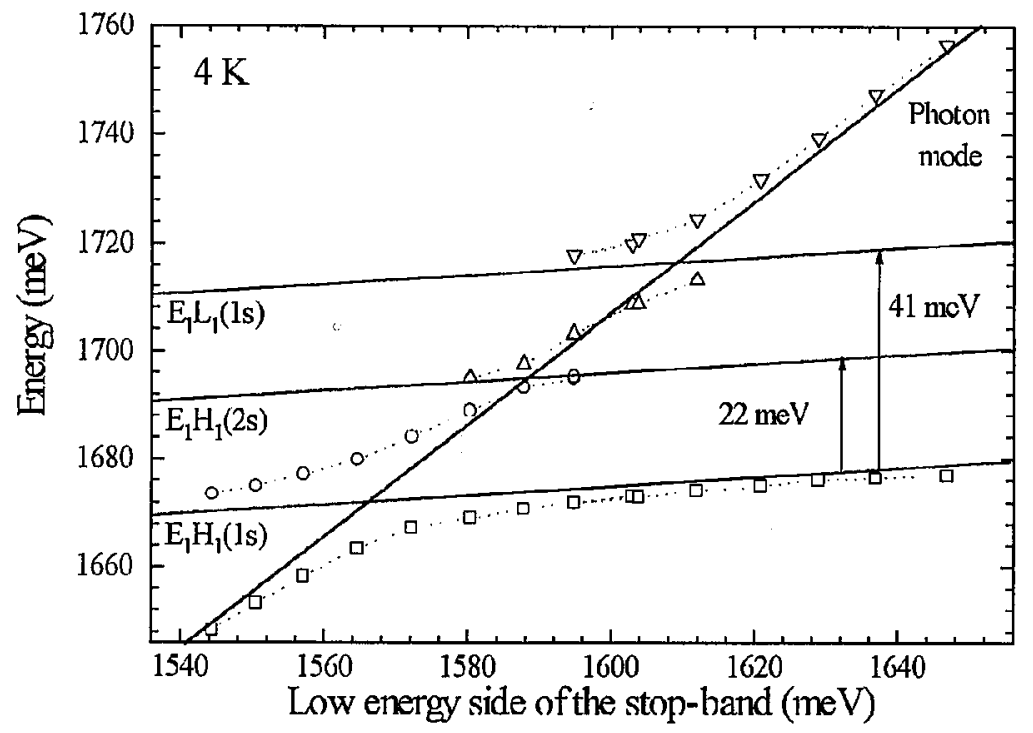

Fig. 4. The different lines pointed in Fig. 3 are plotted versus the low energy side lobe of the Bragg reflectors stop-band. The energy of this lobe is assumed proportional to the sample thickness, i.e. to the bare cavity mode energy.

in the well increases [16]. In that case the oscillator strength derivation is more difficult because the two bare exciton lines are separated by about $3 \mathrm{meV}$ only and the photon mode couples simultaneously to $\mathrm{X}$ and $\mathrm{X}^{+}$. 


\subsection{Temperature effect on the Rabi splitting}

The evolution of the Rabi splitting versus temperature for polaritons built with the fundamental E1H1(1s) exciton is depicted in Fig. 5 for three different samples (S3: three $88 \AA$ QWs in a $\lambda$ cavity, S6: six $50 \AA$ QWs in $3 \lambda / 2$ cavity and S16: sixteen $50 \AA \mathrm{QWs}$ in a $2 \lambda$ cavity). We observe that the splitting is almost constant for low temperature (below $100 \mathrm{~K}$ ) and decreases at higher temperature before vanishing: this is a continuous evolution from strong to weak coupling regime because of an increase in the homogeneous excitonic line width with temperature. The important role of bare states line width for the observation of the strong coupling regime has been pointed out in several papers and the specific effects of homogeneous [17] and inhomogeneous [18] exciton broadening have been reported. To first approximation, the strong coupling disappears when the line widths are of the order of the Rabi splitting expected without broadening.

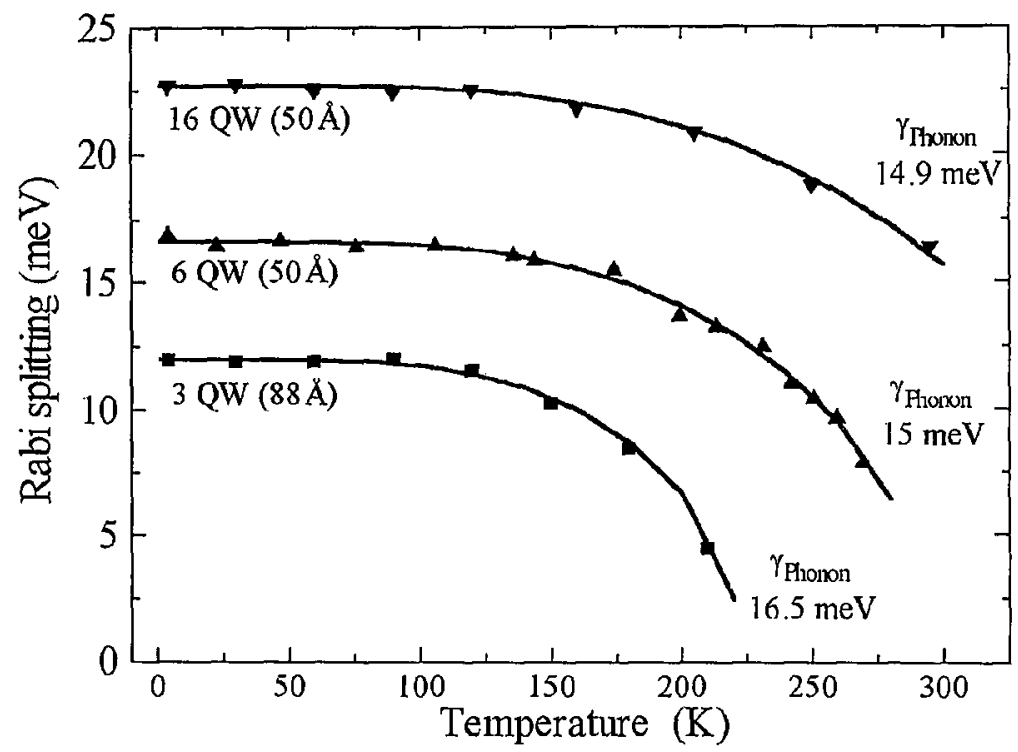

Fig. 5. Temperature effect on the Rabi splitting for three different samples. Solid curves are the result of a model calculation with only one adjustable parameter ( $\left.\boldsymbol{\gamma}_{\mathrm{phonon}}\right)$ characteristic of the exciton-LO phonon interaction (see text).

Two important points may be inferred from Fig. 5. First, it is possible to keep the strong coupling regime at room temperature with CdTe QWs in spite of the high efficiency of exciton-LO phonon scattering in CdTe (see below). Second, the Rabi splittings that we measure at $4 \mathrm{~K}$ are not affected by bare states broadening and reflect directly the exciton-photon coupling as assumed previously $\left(\hbar \Omega_{\mathrm{Rabi}}=2 \mathrm{~V}\right)$. To go beyond this approximation we use a more complete calculation, line width dependent, of the Rabi splitting by Savona et al. [4]:

$\hbar \Omega_{\mathrm{Rabi}}=2 \sqrt{\sqrt{V^{4}\left(1+2 \frac{\Gamma}{\Gamma_{\mathrm{cav}}}\right)^{2}+2 V^{2} \Gamma^{2}\left(1+\frac{\Gamma}{\Gamma_{\mathrm{cav}}}\right)^{2}}-2 V^{2} \frac{\Gamma}{\Gamma_{\mathrm{cav}}}-\Gamma^{2} .}$ 
Note that within this approach the Rabi splitting depends also on the kind of optical measurement (here reflectivity). In the present case, the exciton homogeneous (nonradiative) line width $\Gamma$ increases with temperature due to exciton-phonon scattering. In II-VI semiconductors, it has been shown that the effect of acoustic phonons is negligible compared to that of LO-phonons [19] and then the homogeneous exciton line width increases as the LO-phonon population

$$
\Gamma(T)=\Gamma(0)+\gamma_{\text {phonon }}\left[\exp \left(\hbar \omega_{\mathrm{LO}} / k_{\mathrm{B}} T\right)-1\right]^{-1},
$$

where $\gamma_{\text {phonon }}$ represents the strength of the exciton-phonon coupling. From Eqs. (4) and (5) one gets $\hbar \Omega_{\text {Rabi }}$ as a function of temperature depending on only one unknown parameter: $\gamma_{\text {phonon. }}$ This parameter was adjusted in the calculation of the curves in Fig. 5 to fit the experimental data points. The exciton-phonon coupling is described by $\gamma_{\text {phonon }}=16.5 \mathrm{meV}, 15 \mathrm{meV}$, and $14.9 \mathrm{meV}$ for S3, S6, and S16 respectively.

In sample $\mathrm{S} 6$ and $\mathrm{S} 16, E_{2 s}-E_{1 s}=22 \mathrm{meV}$ is slightly larger than $\hbar \omega_{\mathrm{LO}}$ $(21.3 \mathrm{meV})$. In that case, scattering of radiative exciton toward excited states or dissociation into the electron-hole continuum is not allowed. The exciton states a vailable for scattering are restricted to the $1 s$ exciton dispersion curve. This situation minimizes exciton broadening with temperature. $\gamma_{\text {phonon }}$ increases for larger QWs (S3) with smaller binding energy.

Up to now we have ignored the role of polaritons in the broadening mechanism which is in fact a phonon-polariton scattering [20]. As long as only LO-phonons are involved, with an energy $(21.3 \mathrm{meV})$ clearly larger than $\hbar \Omega_{\mathrm{Rabi}} / 2$, exciton-polaritons are scattered in the exciton-like part of the LPB dispersion curve. The density of final state for the scattering process is then almost the same as for scattering with QW excitons. The situation is totally different from acoustic phonons scattering in III-V semiconductors. From an experimental point of view, the fact that the same quantum wells (50 $\AA$ thick) give the same exciton-phonon coupling factor even in different samples (S6 and S16) with very different polariton dispersion curves justifies that we neglect the role of polaritons in broadening mechanism.

The homogeneous broadening is a limiting factor for cavity polaritons at high temperature, but the decrease in the Rabi splitting with temperature is also an interesting probe for exciton-LO phonon interaction.

\section{Polariton relaxation dynamics}

In this section we present experimental results on the time-resolved $\mathrm{PL}$ from LPB polaritons on a picosecond scale. PL dynamics measurements were performed at low temperature in the linear regime and with non-resonant excitation, either as a function of the detuning or as a function of the number of QWs in the microcavity samples.

The PL dynamics at $k_{\|}=0$ was first studied, for a $1-\mathrm{QW}$ microcavity sample, as a function of the detuning $\Delta E=E_{\text {photon }}-E_{\text {exciton. }}$. We observed a constant decay time of about $120 \mathrm{ps}$ for positive or slightly negative detuning, but for $\Delta E<-5 \mathrm{meV}$ the $\mathrm{PL}$ decay time increases dramatically up to $420 \mathrm{ps}$ (Fig. 6). The high value of those decay times compared to the radiative lifetime of the polariton 
(typically, one picosecond) shows that the decay time of the PL is governed by relaxation process from excitons at large $k_{\|}$to radiative polariton states. The origin of the abrupt variation of the PL decay time is the following. For large $k_{\|}$values, the LPB is nearly dispersionless, i.e. exciton-like, independently of the detuning, but for negative photon-exciton detuning the LPB is photon-like for small $k_{\|}$-values (Fig. 1b). The abrupt variation from an exciton-like to a photon-like dispersion near the resonance results in a bottleneck effect in the relaxation by acoustic phonons [21]. When the LPB becomes photon-like (region of negative curvature on the LPB, Fig. 1b) the relaxation by acoustic phonons is less efficient because the phonons interact only with the decreasing excitonic component of the LPB and the relaxation rate which is proportional to the density of final states decreases when the dispersion slope increases. Moreover, the relaxation from the bottleneck region to the bottom of the LPB is more and more difficult when the photon mode is tuned well below the exciton energy because the scattering rate drastically decreases for acoustic phonon energies larger than $3 \mathrm{meV}$. On the contrary, the emission near $k_{\|}=0$ is easier when the bottom of the LPB is photon-like, but obviously the PL decay time is governed by the slowest step.
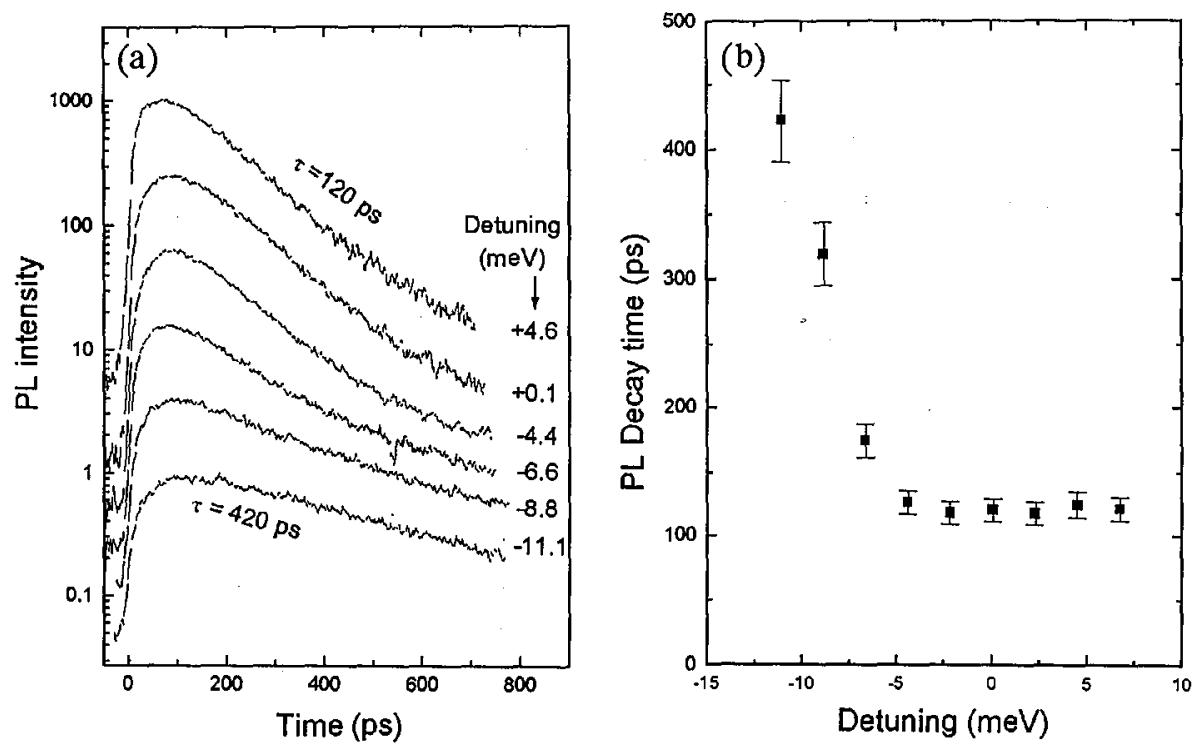

Fig. 6. Time evolution of PL intensity (in arb. units) at the LPB energy for various detuning (a). Decay time versus exciton-photon detuning (b).

Another aspect of the relaxation processes was investigated: in a microcavity with $N$ QWs, the eigenstates of the system consist of $N-1$ purely excitonic (antisymmetric) states [22] and two polariton states built with the symmetric combination of the excitonic states. The $N-1$ nonradiative states (the so-called "dark states") are likely to influence the PL dynamics as they could constitute additional relaxation channels. In order to demonstrate this effect, we grew a series of three identical microcavity samples containing respectively 1,2 , 
and 4 QWs. The position of the QWs in each sample was calculated to keep the same Rabi splitting ( $7 \mathrm{meV}$ ), and therefore the same shape of the dispersion curves, but with different dark states degeneracy. We observed that the 4-QWs sample presents, for $\Delta E=-10 \mathrm{meV}$, a PL decay time twice shorter than the 1-QW sample. This decrease, which is monotonic with the number of QWs, suggests that the dark states participate in the relaxation towards the radiative states located near $k_{\|}=0$.

\section{Stimulated emission of polariton luminescence}

This last section is devoted to stimulated emission of cavity polariton under non-resonant excitation. A nonlinear emission of the lower polariton PL, with a marked threshold-like behavior, is observed in QMC for excitation densities well below exciton screening. In such excitation conditions the strong coupling is hardly affected and the emission, linear or nonlinear, occurs from cavity polariton states [23-25].

We observed the stimulation of cavity polaritons PL in a large series of samples with different $Q W$ s number, different Rabi splitting and for various excitonphoton detuning. The most spectacular effect is observed for the larger number of QWs (16 QWs in a $2 \lambda$ cavity - sample S16), when the cavity mode is exactly resonant with the exciton energy at $k_{\|}=0$. In Fig. 7 , the LPB and UPB energies in the linear regime are given by the reflectivity spectrum and the PL lines obtained under increasing excitation density are shown on the same plot for comparison. The PL spectra are divided by the excitation intensity to detect any nonlinear response. A linear PL emission is observed for excitation densities below $40 \mathrm{~kW} / \mathrm{cm}^{2}$.

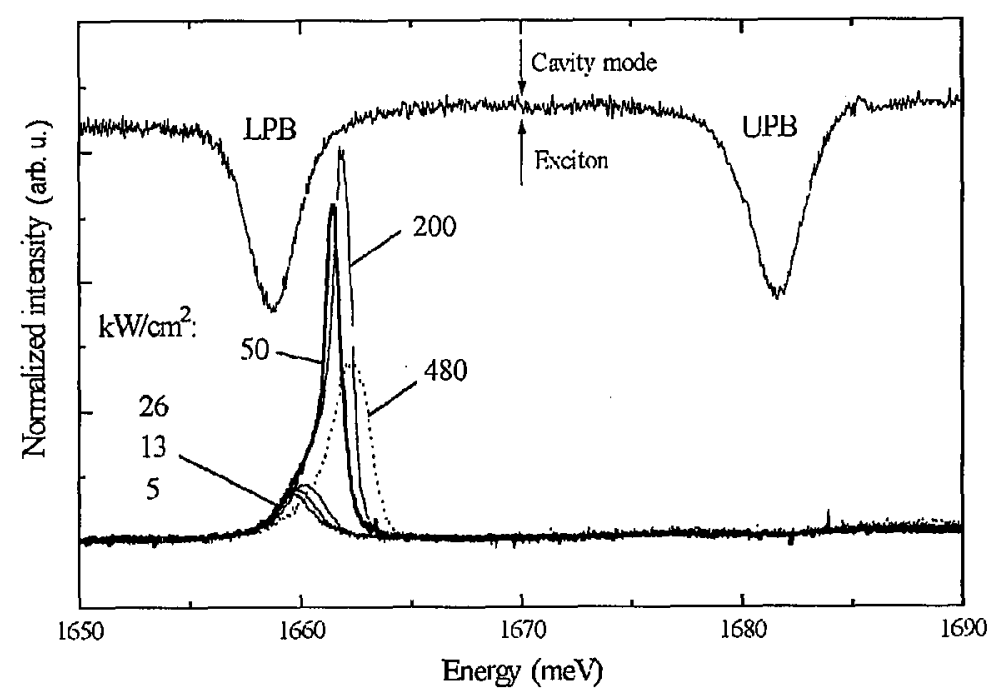

Fig. 7. LPB luminescence $(4.2 \mathrm{~K})$ at exact exciton-photon resonance and under increasing excitation. The PL intensity is divided by the excitation density to evidence a marked nonlinear behavior after $40 \mathrm{~kW} / \mathrm{cm}^{2}$. A reflectivity spectrum (low intensity limit) is given for comparison. 
After this threshold value a sharp line emerges whose intensity increases nonlinearly with excitation. After $200 \mathrm{~kW} / \mathrm{cm}^{2}$ this line broadens and shifts to higher energies before it vanishes. For much higher excitations a new line appears at the energy of the uncoupled cavity mode (not shown). The intensity of this line behaves also nonlinearly after a second threshold of about $10 \mathrm{MW} / \mathrm{cm}^{2}$ which is attributed to the usual laser effect by population inversion of the electron-hole plasma.

At the onset of the nonlinear emission the spectral position of the PL line is almost identical to the LPB energy observed under low excitation densities. The slight shift with respect to the linear emission is one order of magnitude smaller than the Rabi splitting ( $23 \mathrm{meV}$ ) measured on the reflectivity spectrum, and although the small energy shift may be explained by a slight decrease in the oscillator strength, the nonlinear emission occurs on the LPB line well before the transition to the weak coupling regime. This is confirmed by the observation of $\mathrm{PL}$ at the uncoupled cavity mode for much larger excitations. Hence, the nonlinear emission that we observe cannot be explained by fermionic electron-hole correlations after collapse of the strong coupling regime as suggested in [26]. We are not either in the same conditions as in Ref. [27] where laser emission in a microcavity arises from population inversion of localized excitons. We believe that the theoretical approach of exciton-polariton stimulation proposed by Imamoglu et al. [28] is more suitable to our observations: the relaxation from excitons created at high energy to the bottom of the LPB by exciton-phonon or exciton-exciton scattering may be strongly enhanced by the final-state stimulation effect. As long as exciton-polaritons may be considered as bosons, the relaxation rate from a population reservoir above the bottleneck region of the LPB to the bottom of the LPB

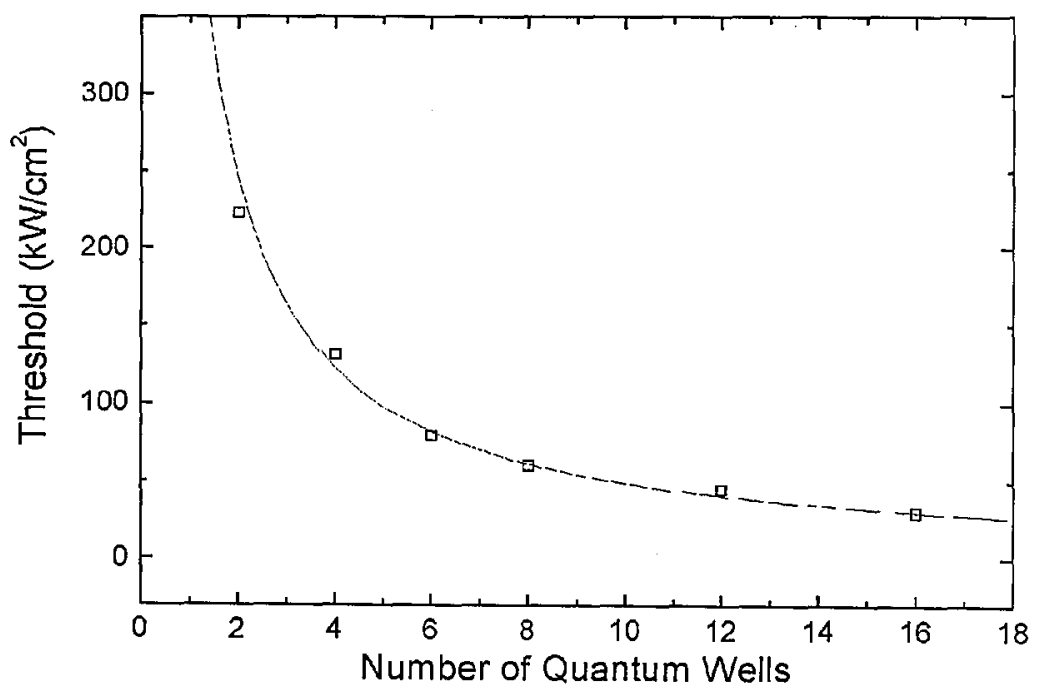

Fig. 8. Polariton (LPB) stimulation thresholds for various $Q M C$ samples plotted versus the number of QWs in each sample. 
is proportional to $N_{\text {reservoir }}\left(N_{\mathrm{LPB}}+1\right)$. Consequently, any relaxation process will be stimulated if $N_{\text {LPB }}>1$. This effect is only possible if the exciton density is not too high so that they could be considered as bosons. The final state stimulation is also consistent with the observation that the threshold of the LPB luminescence stimulation exhibits a strong increase with negative detuning: when the energy difference between the bottleneck region and the LPB at $k_{\|} \approx 0$ increases, the critical final state density for stimulation is more difficult to reach except when the energy difference is exactly one LO-phonon. For this last situation we observed that the stimulation threshold presents a marked decrease.

Figure 8 shows the evolution of the LPB nonlinear emission threshold at resonance for a large series of microcavity samples containing from $N=2$ to $16 \mathrm{QWs}$ with different cavity length or the Rabi splitting. Whatever the various parameters of those samples, the stimulation thresholds are exactly in the inverse ratio of the number of QWs. Such a behavior is far from a usual population inversion in QWs: the point here is not to invert the population of $N$ QWs but, on the contrary, $N$ QWs contribute to the population buildup of one polariton state.

\section{Conclusion}

In this paper we review the main results obtained in the last two years on the physics of cavity polaritons in CdTe-based microcavities. After general considerations on $2 \mathrm{D}$ polaritons and introduction of this concept using the two coupled oscillator model, we showed, in the linear regime, that the strong coupling may be an efficient probe for excitons in CdTe QWs. The exciton-LO phonon scattering was studied and a continuous transition from strong to weak coupling regime with increasing temperature was observed. Nevertheless, a Rabi splitting of $16 \mathrm{meV}$ remains up to room temperature in a purposely designed sample.

Our results on cavity polariton luminescence are strongly related to polariton relaxation phenomena. Time-resolved PL studies showed that a bottleneck effect is present in the relaxation along the LPB. The relaxation toward $k_{\|}=0$ is more and more difficult when the photon energy is tuned below the exciton energy. Under increasing excitation density a strongly nonlinear emission is observed after a threshold value. This emission occurs in the strong coupling regime from the LPB state. This nonlinear effect is very different from a usual laser emission and has been interpreted as stimulation of the relaxation processes from a reservoir population above the bottleneck toward the bottom of the LPB dispersion. This is a consequence of the bosonic nature of cavity-polaritons.

This work was completed within the CNRS-CEA joint group "Nanophysique et semiconducteurs" in Grenoble.

\section{References}

[1] C. Weisbuch, M. Nishioka, A. Ishikawa, Y. Arakawa, Phys. Rev. Lett. 69, 3314 (1992).

[2] S. Jorda, Phys. Rev. B 50, 18690 (1994); S. Jorda, Phys. Rev. B 51, 10185 (1995).

[3]. V. Savona, Z. Hradil, A. Quattropani, P. Schwendimann, Phys. Rev. B 49, 8774 (1994). 
[4] V. Savona, L.C. Andreani, P. Schwendimann, A. Quattropani, Solid State Commun. 93, 733 (1995).

[5] S. Pau, G. Björk, J. Jacobson, H. Cao, Y. Yamamoto, Phys. Rev. B 51, 14437 (1995).

[6] M.S. Skolnick, T.A. Fisher, D.M. Whittaker, Semicond. Sci. Technol. 13, 645 (1998).

[7] V. Savona, C. Piermarocchi, A. Quattropani, P. Schwendimann, F. Tassone, Phase Transit. 68, 169 (1999).

[8] P. Yeh, Optical Waves in Layered Media, Wiley, New York 1988.

[9] E.L. Ivchenko, A.I. Nesvizhskii, S. Jorda, Phys. Solid State 36, 1156 (1994).

[10] L.C. Andreani, Phys. Lett. A 192, 99 (1994).

[11] H. Davies, Proc. Instn. Elect. Engrs IV 101, 209 (1954).

[12] Y. Merle d'Aubigné, H. Mariette, N. Magnea, H. Tuffigo, R.T. Cox, G. Lentz, Le Si Dang, J.-L. Pautrat, A. Wasiela, J. Cryst. Growth 101, 650 (1990).

[13] R. André, Le Si Dang, J. Appl. Phys. 82, 5086 (1997).

[14] W.T. Masselink, P.J. Pearah, J. Klem, C.K. Peng, H. Morkoc, G.D. Sanders, Yia-Chung Chang, Phys. Rev. B 32, 8027 (1985).

[15] R. André, D. Heger, Le Si Dang, Y. Merle d'Aubigné, J. Cryst. Growth 184/185, 758 (1998).

[16] T. Brunhes, R. André, A. Arnoult, J. Cibert, A. Wasiela, to be published in Phys. Rev. $B$.

[17] R. Houdré, R.P. Stanley, U. Oesterle, M. Ilegems, C. Weisbuch, Phys. Rev. B 49, 16761 (1994).

[18] S. Pau, G. Björg, H. Cao, E. Hanamura, Y. Yamamoto, Solid State Commun. 98, 781 (1996).

[19] N.T. Pelekanos, J. Ding, M. Hagerott, A.V. Nurmiko, H. Luo, N. Samarth, J.K. Furdyna, Phys. Rev. B 45, 6037 (1992); N.T. Pelekanos, H. Haas, N. Magnea, H. Mariette, A. Wasiela, Appl. Phys. Lett. 61, 3154 (1992).

[20] V. Savona, C. Piermarocchi, Phys. Status Solidi A 164, 45 (1997).

[21] F. Tassone, C. Piermarocchi, V.Savona, A. Quattropani, P. Schwendimann, Phys. Rev. B 56, 7554 (1997).

[22] G. Panzarini, L.C. Andreani, Phys. Rev. B 52, 10780 (1995).

[23] J. Bleuse, F. Kany, A.P. de Boer, P.C.M. Christianen, R. André, H. Ulmer-Tuffigo, J. Cryst. Growth 184/185, 750 (1998).

[24] Le Si Dang, D. Heger, R. André, F. Boeuf, R. Romestain, Phys. Rev. Lett. 81, 3920 (1998).

[25] P. Senellart, J. Bloch, Phys. Rev. Lett. 82, 1233 (1999).

[26] M. Kira, F. Jahnke, S.W. Koch, J. Berger, D. Wick, T. Nelson, Jr., G. Khitrova, H. Gibbs, Phys. Rev. Lett. 79, 5170 (1997).

[27] X. Fan, H. Wang, H.Q. Hou, B.E. Hammons, Phys. Rev. B 56, 15256 (1997).

[28] A. Imamoglu, R. Ram, Phys. Lett. A 214, 193 (1996); A. Imamoglu, R. Ram, S. Pau, Y. Yamamoto, Phys. Rev. A 53, 4250 (1996). 